\title{
A Channel Allocation Scheme with Dynamic Priority for Wireless Mobile Networks
}

\author{
Huei-Wen Ferng, Hsin-Jung Lin, Wei-Chung Teng, Yi-Chou Tsai, and Cheng-Ching Peng \\ Department of Computer Science and Information Engineering \\ National Taiwan University of Science and Technology, Taipei 106, Taiwan \\ Email: hwferng@mail.ntust.edu.tw
}

\begin{abstract}
A channel allocation scheme based on the technique of guard channel employing dynamic priority with three queues (DPTQ) is proposed in this paper. DPTQ differs from most of previously proposed schemes in the literature which focused on reducing forced-termination for handoff calls only. It tries to reduce blocking/dropping probabilities for new calls and data calls with tolerable forced-termination probability for the handoff call in wireless networks. This scheme is especially suitable for the operation in a hot spot in which less handoff calls may occur. In order to compare our scheme with the scheme called dualthreshold bandwidth reservation (DTBR) previously proposed in the literature in which no queue is employed, three queues are endowed with DTBR to form dual-threshold with three queues (DTTQ). Through numeral comparisons, we show that DPTQ outperforms DTTQ in blocking/dropping probabilities for new calls and data calls with a bit increase of the forced-termination probability for handoff calls.
\end{abstract}

\section{INTRODUCTION}

The wireless mobile networks use air as communication interface in which only limited radio frequency resources are available. As the number of users and mobile devices continue remarkable growth in recent years, it becomes more and more important to develop a sophisticated mechanism for resource allocation with optimal usage efficiency. One major objective on this issue is to achieve low call-incompletion probability. Channel allocation is the key technique for this objective.

Many schemes focusing on channel allocation of new calls and handoff calls have been reported in the literature, e.g., [1], [2], [3], [4], [5]. The simplest non-prioritized scheme treats both new calls and handoff calls equally, that is to say, every arriving call is allocated a channel if idle channels are available or it is blocked otherwise. The channel reservation scheme separates channels into two groups: one group dedicated to handoff calls and the other group used as common channels for all calls. Another kind of schemes which is known as DTBR [5] reserves the dedicated group to both new calls and handoff calls and confines data calls to the common channel group with the lowest priority. At last, queues for calls may be utilized at some designs, e.g., [4], [6], [7], to decrease call blocking probabilities.

Some systems further utilize channel borrowing in their designs, e.g., [8], [9], [10], [11]. Channel borrowing is usually used in fixed channel assignment (FCA) schemes. In these systems, every cell is assigned the same number of channels at the first time. When some cells runs out of idle channels due to high traffic load or other reasons, it is allowed these cells to temporarily borrow idle channels from adjacent cells if available. Lee and Cho [9] use the feature that a mobile station may hold two channels within the handoff area. They use channel borrowing to increase system service capacity. A restriction in channel borrowing schemes is that the borrowed channel must be locked in the reusable cells. Zhang and Yum [8] introduced a directional locking method which effectively decreases the number of locked channels and thus greatly improves total frequency usage efficiency, especially for the case that most cells have high traffic load and the chain action of borrowing channels from adjacent cells happens. Some other systems give handoff calls the right to preempt the serving channels. In these systems, an arriving handoff call occupies a channel from some other new call or data call when no idle channel is available in the cell. The interrupted calls then stop and wait in queue or are forced to terminate. Some designs allow the probability of channel preemption by handoff calls adjustable, e.g., [6], [7].

In the literature, most channel allocation schemes have laid stress on the forced-termination probability of handoff calls. To reach lower forced-termination probabilities, new calls are usually sacrificed, thus resulting in high blocking probabilities. For data calls, due to the feature of their tolerance to longer waiting time, the lowest priority is always set to them. In most cases, the above policy is appropriate. But this policy may not be suitable under some conditions like hot spot in which most mobile stations seldom move or even never leave the current area. So there are rare handoff calls in the hot spot. The most famous example for such situations is New Year's countdown. People in parties form hot spots one by one. Thus, policies weighting handoff calls are no longer suitable for such situations.

In this paper, we propose a flexible channel allocation scheme. It is adjustable to various conditions to achieve better performance. Even in the hot spot, our scheme shows lower blocking/dropping probabilities for new calls and data calls. Although the adjustment may cause higher forced-termination probabilities for handoff calls, it is acceptable if the probability falls below $10^{-3}$ for general cases [5].

The rest of the paper is organized as follows. In Section II, we demonstrate the channel allocation scheme. Section III shows numerical results and performance evaluation. Finally, 
Section IV concludes the paper.

\section{Channel Allocation Scheme}

\section{A. Model Assumptions}

To simplify our model, we only consider homogeneous cells in which a fixed number of channels $C$ is assumed. Thus, we only need to observe single cell here. We call this observing cell the target cell. In this paper, data transmission is categorized into three types: handoff calls, new calls, and data calls. The channel allocation scheme for each cell is illustrated in Fig. 1. The $C$ channels of each cell are further divided into two parts. The first part include $C_{c}$ common channels shared by all three types of calls. In this figure, point $a$ stands for a threshold for the number of data calls. When the number of data calls reaches point $a$, the target cell stops allocating channels to the subsequent data calls. The second part has $C-C_{c}$ channels shared by both handoff calls and new calls. We assume the arrival rate of handoff calls is $\lambda_{h}$, the arrival rate of new calls is $\lambda_{n}$, and the arrival rate of data calls is $\lambda_{d}$, respectively, with their service/departure rates $\mu_{h}, \mu_{n}$, and $\mu_{d}$, respectively. In addition, there are three queues to buffer these three types of calls with buffer size $Q_{h}, Q_{n}$, and $Q_{h}$, respectively. Usually $Q_{h}$ and $Q_{n}$ are set to similar values. So we let $Q_{h}=Q_{d}=Q_{d}$ in this paper. Since our approach permits dynamic priorities to both handoff calls and new calls, we use the term DPTQ, i,e. dynamic priority with triple queues for our approach in this paper. Finally, we ignore the cases due to timeout of calls in queues.

\section{B. Details of the Scheme}

The procedure for call arrival is described as follows. Check available channels from the part of common channels at first when a handoff call or a new call arrives. If there is no idle channel available, then check from the non-common channel part. Allocate an idle channel to the call, if available; otherwise the call enters its queue. In case the queue is already full, the call is blocked or forced terminated. When a data call arrives, check also available channels from the common channel part. If the number of currently used channels $C_{u}$ is less than $C_{c}$, then at least one idle channel is available for the data call. If $C_{u}$ is not less than $C_{c}$ but less than $C$ and if the number of channels used by data calls $N_{d}$ is less than $C_{c}$, then channel reallocation is applied to yield idle channels from the common channel part since there must be handoff calls or new calls holding common channels. For the case of $N_{d}=C_{c}$, any subsequent calls are then pushed into the queue or get dropped if the data call queue is full.

The procedure of channel release is similar to that of channel allocation. If the handoff call queue and new call queue are empty but the data call queue is not empty, then a queued data call gets this released channel only when $N_{d}<C_{c}$ (of course, channel reallocation may be used). For the case of the handoff call queue or the new call queue is not empty, this released channel is immediately allocated to the queued call. However, when both handoff and new call queues are not empty, priorities should be examined based on queue lengths. Here, we set a threshold $T_{h}$ to make dynamic priorities possible. In general, we give handoff calls higher priorities over new calls if the queue length difference of the new call queue and handoff call queue is less than $T_{h}$; otherwise, we let new calls have higher priorities. The threshold value $T_{h}$ then becomes a tunable parameter to adapt system conditions.

We now elaborate on the modified DTBR scheme, i.e., DTTQ as contrast to DPTQ. Its operation is illustrated in Fig. 2. In DTBR, two thresholds are employed: data call threshold and new call threshold. When the number of used channels reaches the data call threshold, subsequent data calls are dropped; when the number of used channels further reaches the new call threshold, subsequent new calls are also blocked. To compare DPTQ with DTBR under the same basis, we add three queues to the DTBR model, i.e., handoff call queue, new call queue, and data call queue to form DTTQ. In DTTQ, priorities from the highest to the lowest are handoff calls, new calls, then data calls.

\section{Simulation Results And Performance COMPARISON}

In this section, we demonstrate numerical results by various parameters to get performance of DPTQ and DTTQ. Parameters used in the simulation are set as follows: $C=10, C_{c}=4$, $\mu_{h}=1, \mu_{n}=1, \mu_{d}=10, \lambda_{h}=3, \lambda_{n}=3, \lambda_{d}=5, T_{h}=1$, $Q_{h}=Q_{d}=Q_{d}=5$ (for simplicity).

Fig. 3 shows the forced-termination probability of handoff calls, blocking probability of new calls, and dropping probability of data calls under different traffic load. It is obvious that handoff calls (with the highest priority) always shows the lowest probability; new calls have the second lowest probability; and then the data calls. Fig. 4 gives average delay times under different traffic load with trends similar to Fig. 3.

Fig. 5 is the channel idle probability which is defined as the probability that a released channel cannot be used by any queued calls. When the handoff call and new call load increases proportionally to the system traffic load, the channel idle probability decreases gradually as shown in this figure.

Fig. 6 gives changes of forced-termination probability of handoff calls and blocking probability of new calls and dropping probability of data calls for different threshold values $T_{h}$. We notice that smaller $T_{h}$ is, easier new calls get service and vice versa for handoff calls. Since data calls are independent of the threshold, the dropping probability of data calls keeps constant no matter how $T_{h}$ changes.

Fig. 7 demonstrates the effect of adjusting the number of common channels $C_{c}$. Since the value of $C_{c}$ directly relates to behavior of data calls, we let $\lambda_{d}=20$ (a bit bigger) to enlarge the difference. We know that a bigger $C_{c}$ allows data calls to have more available channels. Thus, it is intuitive that average delay time for data calls drops as shown in Fig. 7. On the other hand, we observe a bit growth on forced-termination probability and blocking probability.

In Figs. 9-12, we compare DPTQ with DTTQ. We try to simulate the hot spot condition by setting $\lambda_{h}=2.5$ and $\lambda_{n}$ in the range of 2 to 6 . Fig. 9 shows the handoff call 
forced-termination probability for different values of $T_{h}$ and DTTQ under different traffic load. In contrast with DTTQ which always gives handoff calls the highest priority, forcedtermination probabilities in DPTQ grows rapidly as the traffic load goes up. However, all probabilities are below $10^{-3}$ (which may be set as an acceptable value). On the other hand, we observe larger improvements in blocking probability of new calls as compared to DTTQ. In fact, DPTQ shows $81 \%$ improvement over DTTQ at the highest traffic load. Since the behavior of data calls is independent of $T_{h}$, so we choose $T_{h}=2$ for Fig. 11. We notice that our scheme shows $74 \%$ improvement over DTTQ at the high traffic load. In Fig. 12, we see that change of $T_{h}$ does not affect the channel idle probability much. In DPTQ, the channel idle probability is affected only by data calls. From Fig. 5, we know that the dropping probability of data calls is independent of $T_{h}$. So curves of different $T_{h}$ almost overlap as shown in Fig. 12. On the contrary, the channel idle probability in DTTQ can be caused by both data calls and new calls. At last, The improvement on the channel idle probability over DTTQ can reach to $99 \%$.

\section{CONCLUSIONS}

In this paper, we propose a channel allocation scheme with dynamic priority called DPTQ. From simulation results, we know that such a scheme benefits by flexibility and can adapt to system conditions by adjusting the threshold $T_{h}$. Of course, it is suitable for the hot spot in which there is rare handoff traffic. Moreover, we also compare DPTQ with a previously proposed scheme DTTQ. We demonstrate that the improvement in performance can reach to $99 \%$. Thus, DPTQ is definitely outperforms DTTQ and is a promising and flexible channel allocation scheme.

\section{REFERENCES}

[1] A. Xhafa and O. K. Tinguz, "Performance of delay-dependent queuing of handoff requests with 2 and 3 priority classes in PCS," in Proc. IEEE VTC '01, pp. 946-950, 2001.

[2] A. Xhafa and O. K. Tinguz, "A new queuing scheme for handoff in $3 \mathrm{G}$ wireless networks," in Proc. IEEE VTC '01, pp. 738-742, 2001.

[3] W. K. Lai, Y. J. Jin, H. W. Chen, and C. Y. Pan, "Channel assignment for initial and handoff calls to improve the call-completion probability," IEEE Trans. Veh. Technol., vol. 52, pp. 876-890, July 2003.

[4] J. Chen and D. Cao, "Performance improvement of prioritized handoff schemes in multi-service mobile cellular systems." in Proc. IEEE Canadian Conf. on Electrical and Computer Engineering '02, vol. 3, pp. 13471351, 2002

[5] B. Li, L. Li, B. Li, and X. Cao, "On handoff performance for an integrated voice/data cellular system," Wireless Networks, vol. 9, pp. 393-402, 2003.

[6] J. Wang, Q. A. Zeng, and D. P. Agrawal, "Performance analysis of a preemptive and priority reservation handoff scheme for integrated servicebased wireless mobile networks," IEEE Trans. Mobile Computing, vol. 2, pp. 65-75, Jan.-March 2003.

[7] Q. A. Zeng and D. P. Agrawal, "Modeling and efficient handling of handoffs in integrated wireless mobile networks," IEEE Trans. Veh. Technol., vol. 51, pp. 1469-1478, Nov. 2002.

[8] M. Zhang and T.-S. P. Yum, "Comaprisons of channel-assignment strategies in cellular mobile telephone systems," IEEE Trans. Veh. Technol., vol. 38, pp. 211-215, Nov. 1989.

[9] D. J. Lee and D. H. Cho, "Performance analysis of channel-borrowing handoff scheme based on user mobility in CDMA cellular systems," IEEE Trans. Veh. Technol., vol. 49, pp. 2276-2285, Nov. 2000.
[10] D. J. Lee and D. H. Cho, "Channel-borrowing handoff scheme based on user mobility in CDMA cellular systems," in Proc. IEEE ICC '00, vol. 2, pp. 685-689, June 2000.

[11] K. L. Yeung, T.-S. P. Yum, and M. M. Choy, "Prioritized handoff strategies using channel borrowing-based dynamic channel assignment," in Proc. IEEE PIMRC '96, pp. 1230-1234, Oct. 1996.

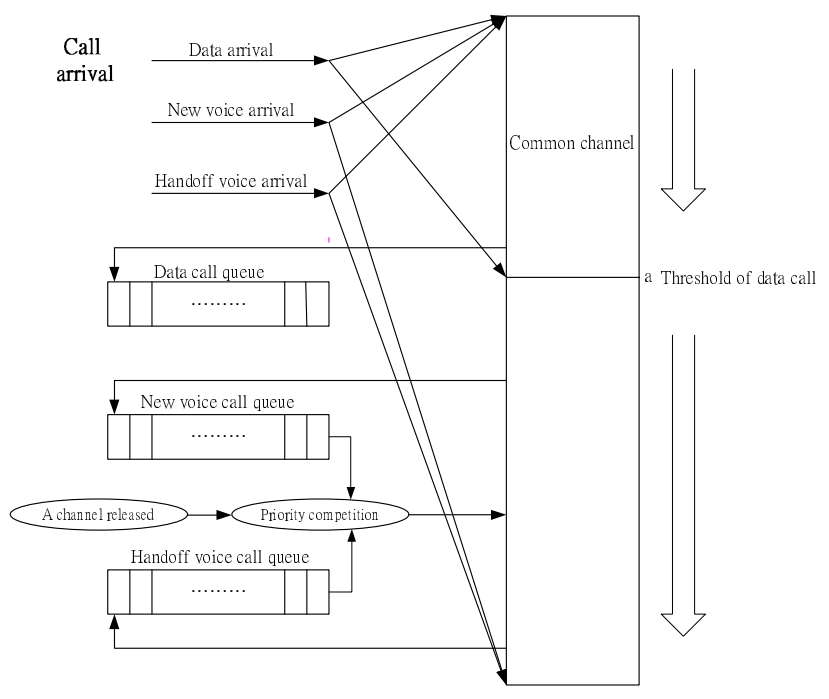

Fig. 1. Channel allocation for DPTQ.

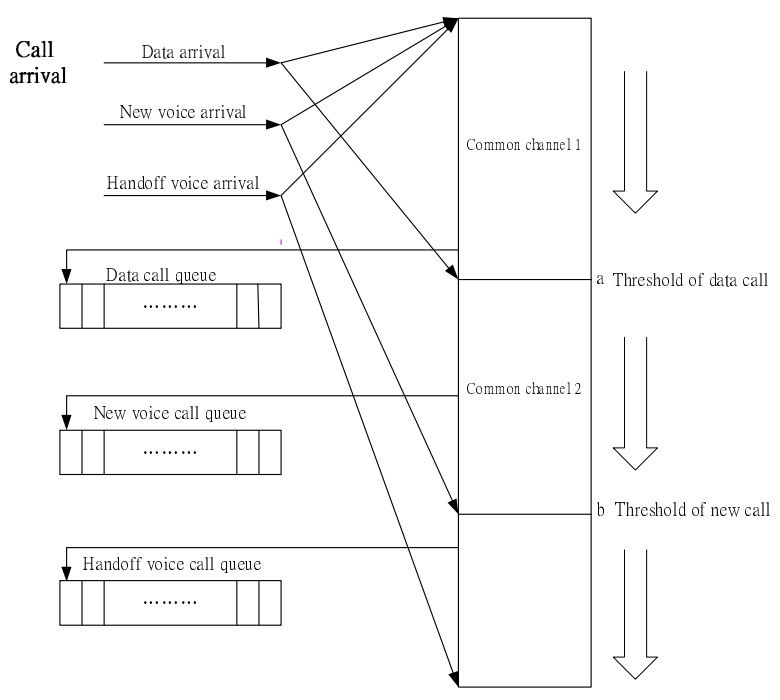

Fig. 2. Channel allocation for DTTQ. 


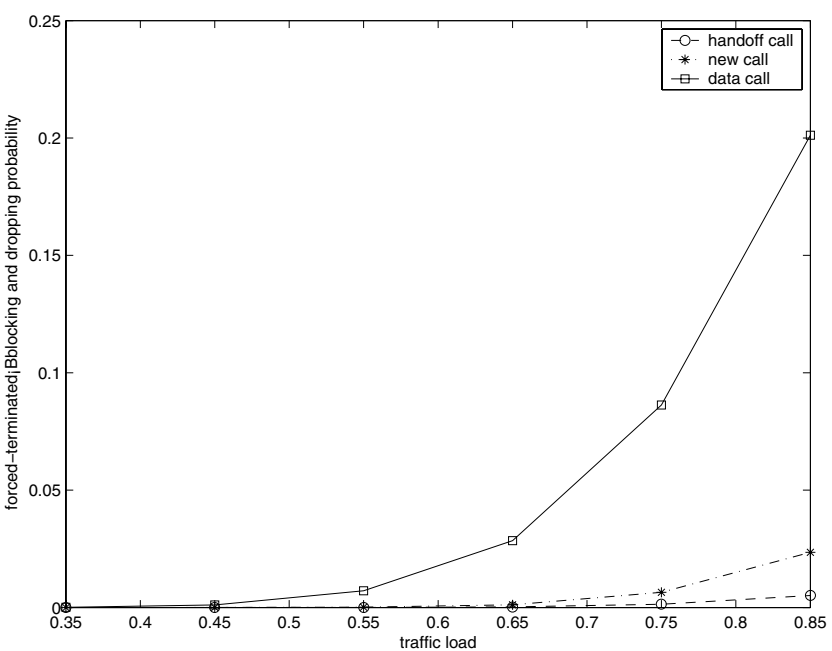

Fig. 3. The forced-termination, blocking, and dropping probabilities of DPTQ for handoff, new, and data calls under different traffic load.

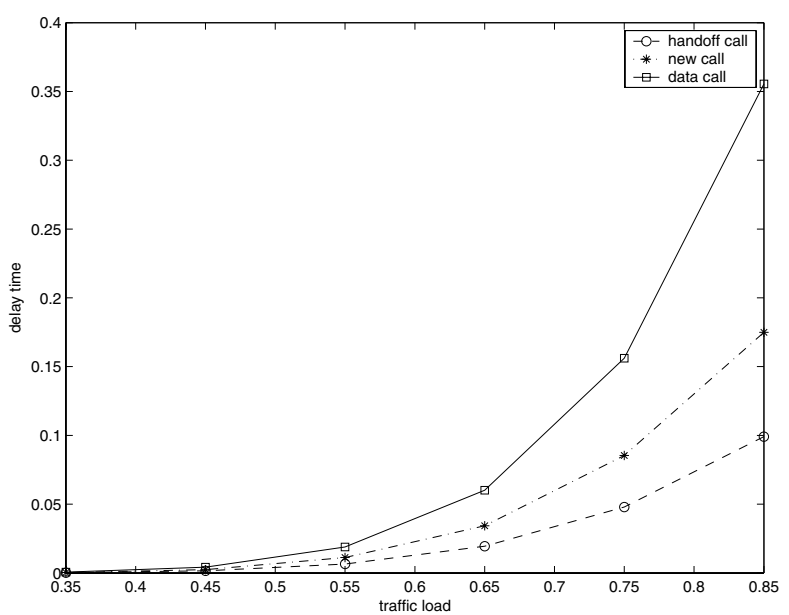

Fig. 4. Average delays for handoff, new, and data calls under different traffic load.

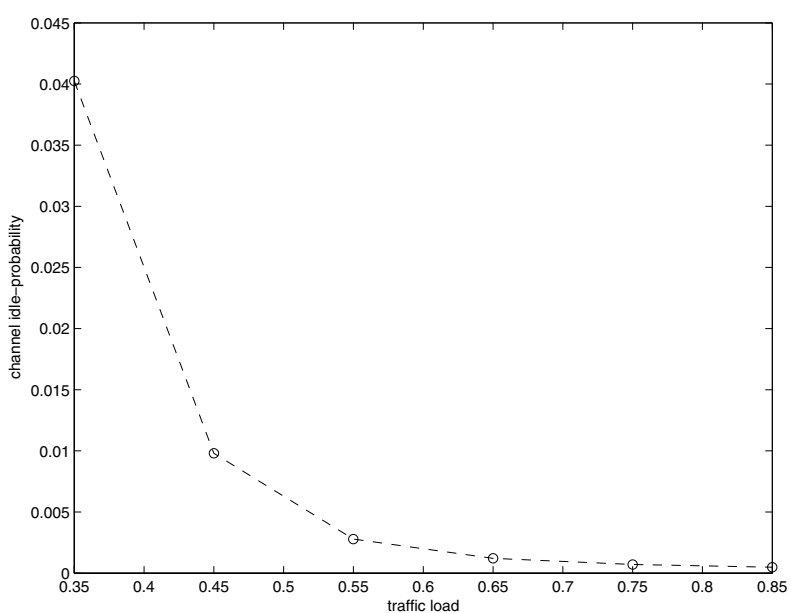

Fig. 5. The channel idle probability under different traffic load.

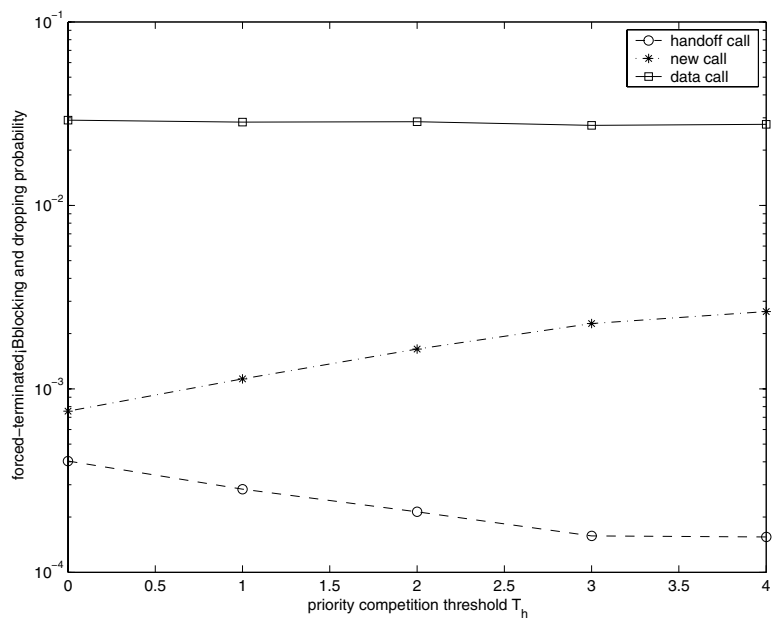

Fig. 6. The forced-termination, blocking, and dropping probabilities for handoff, new, and data calls under different threshold values of $T_{h}$.

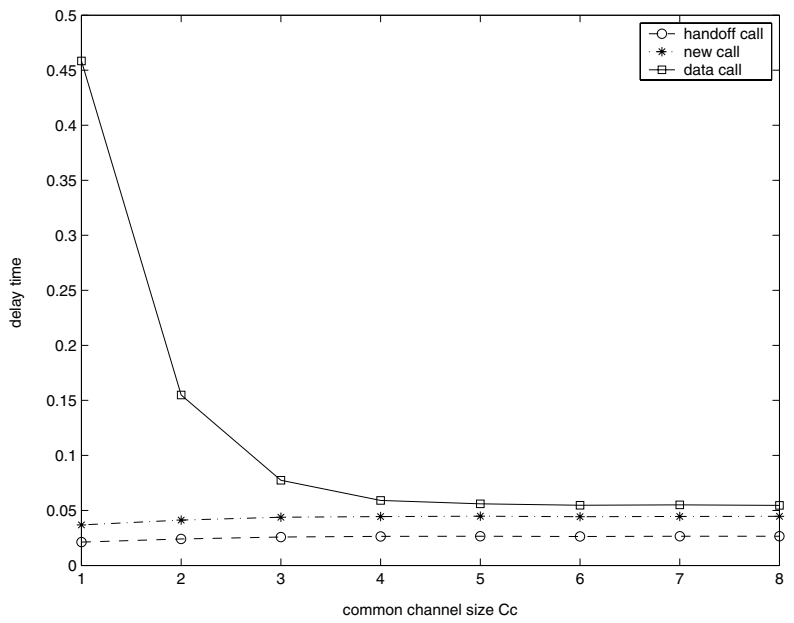

Fig. 7. Average delays for handoff, new, and data calls under different numbers of common channels $C_{c}$.

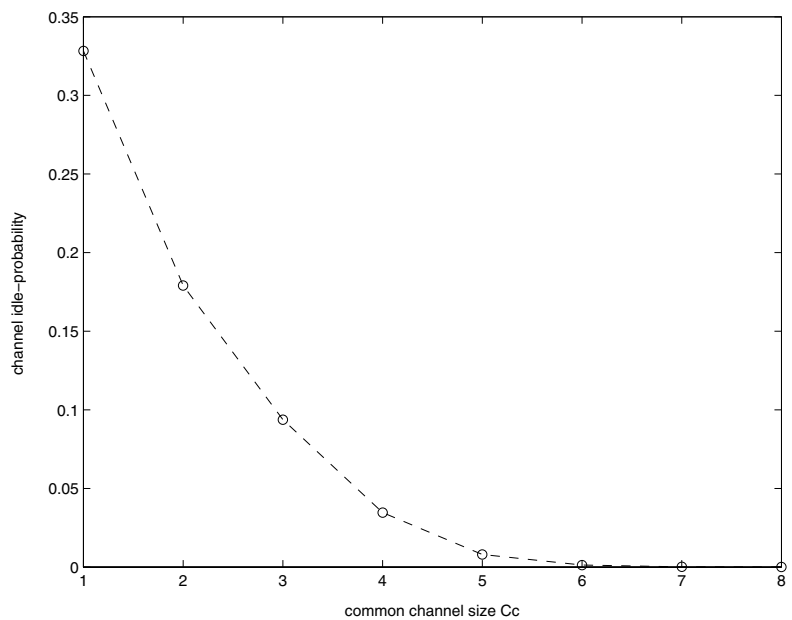

Fig. 8. The channel idle probability under different values of $C_{c}$. 


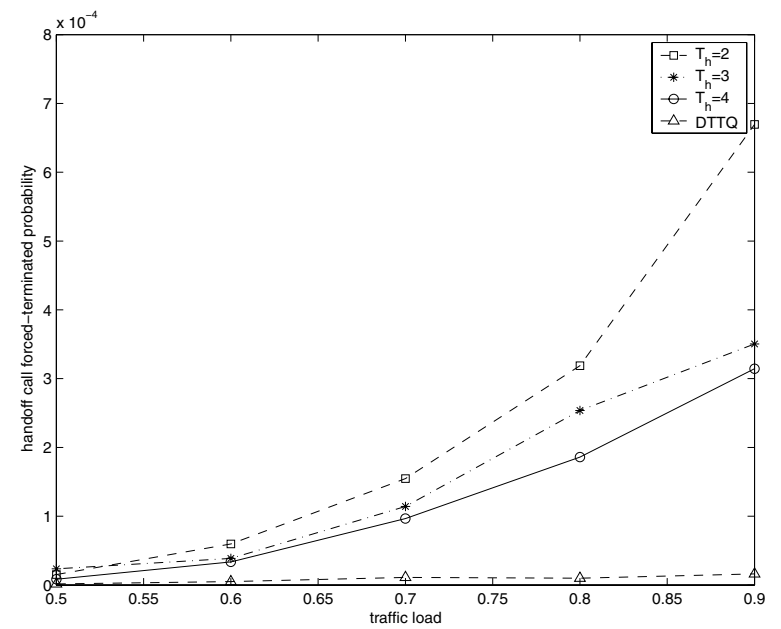

Fig. 9. The handoff call forced-termination probability for DPTQ with different $T_{h}$ and DTTQ udner different traffic load.

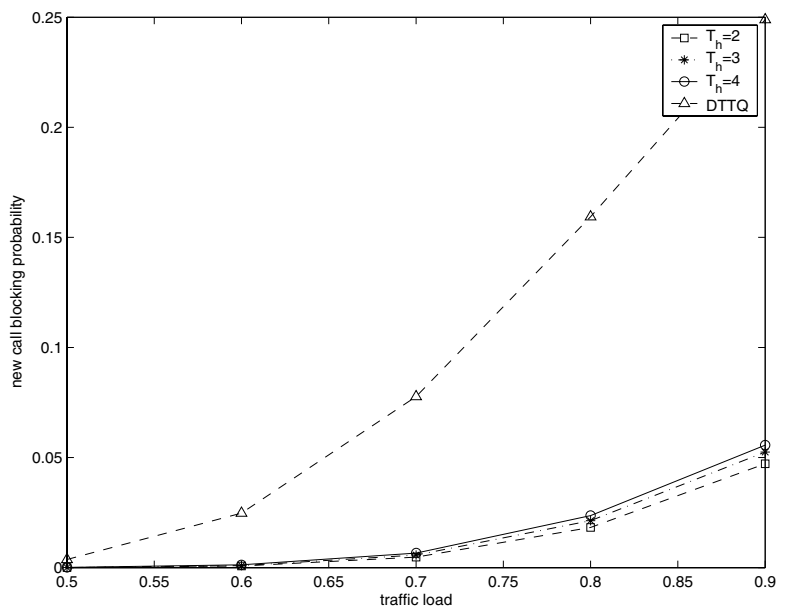

Fig. 10. The new call blocking probability for DPTQ with different $T_{h}$ and DTTQ udner different traffic load.

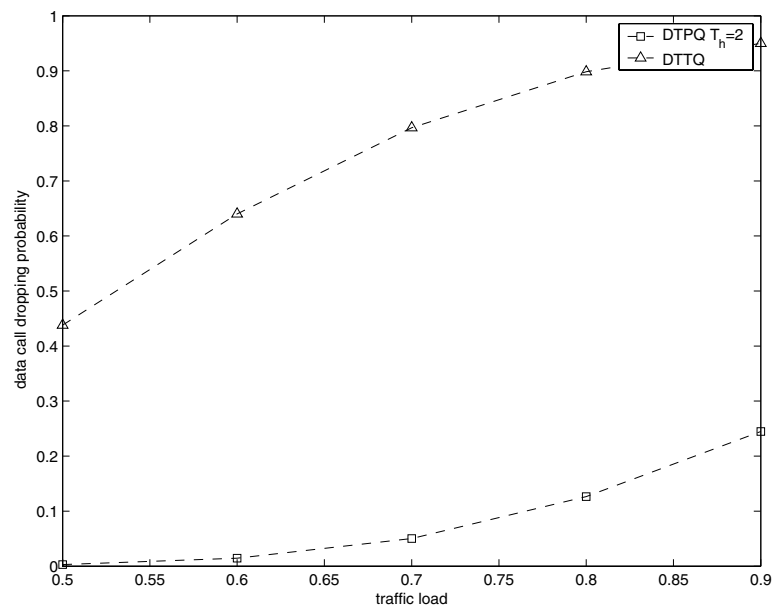

Fig. 11. The data call dropping probability for DPTQ with $T_{h}=2$ and DTTQ udner different traffic load.

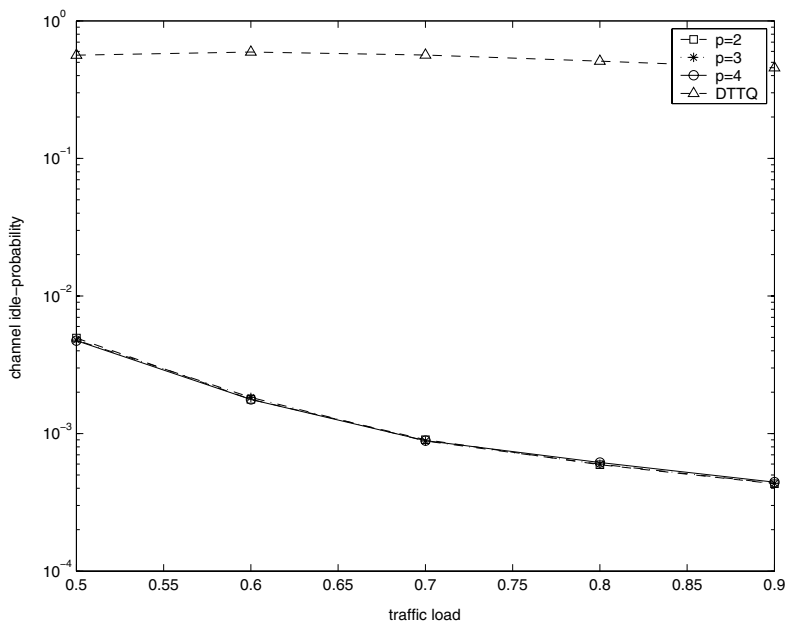

Fig. 12. The channel idle probability for DPTQ with different $p=T_{h}$ and DTTQ udner different traffic load. 